\title{
Polyethylene Gel Volume Fraction (Cross-linked Percentage) Measurement Using DSC Technique
}

\author{
Shouresh Safaei \\ Ankara, Turkey
}

\begin{abstract}
In this paper, the measurement of the crosslinking percentage (gel volume fraction) of polyethylene materials by using DSC technique is presented. To obtain the calibration curve, the networked samples of ASTMD 2765-01 corresponding to the curves of pure melt samples at the start of the reaction were used. The resulting calibration curve was used to predict the percentage of polyethylene cross-linked in a continuous process, and these results were compared with those obtained from D 2765-01, which resulted in excellent agreement between the two states. This technique is faster and cheaper and requires less raw material, the product does not release any harmful substances and is ASTM correct.
\end{abstract}

Keywords: PEO; DSC; gel; cross-link; gel-volume

\section{INTRODUCTION}

They usually obtain a volume fraction of polyethylene using ASTM D 2765-01, which involves storing polyethylene powders in Deca Hydro naphthalene for 6 hours or in xylene for 12 hours, which requires a large workforce and industry. It takes a lot of time, besides they require the use of hazardous materials $[1,2,3,4]$. In this paper, the volume measurement of polyethylene gel has a filler with the proposed method. This method uses the DSC to find peaks of molten polyethylene at the time of use[5,6,7]. he does. The difference between the peaks of the pure melt sample at the onset of the reaction and the peaks of the melt sample can give the percent of crosslinking[8]. The DSC sample is easily prepared by scraping a thin layer of material and punching the sample between the punch papers. There is no need to grind or puncture the specimen so preparation of the specimen is not time-consuming $[9,10,11]$. The specimen is placed in a DSCspecific aluminum foil and there is no need for another specimen container. And the sample is heated at a constant heating rate, which is the program defined in the DSC. It is time[12].

\section{EXPERIMENTAL}

The practical part is straightforward and easy to apply to standard DSC practical structures. This section includes sample preparation and partial analysis of results. Samples of industrial pipe-filled polyethylene are made from Mercury Plastic Inc. These samples were irradiated in an RDI accelerator. The Vixan constant acceleration parameters were used for polyethylene samples. The third sample is three times .... Then 7 different irradiated samples corresponding to 7 samples having different percentages of grids were constructed. Then each sample was divided into two parts and one part was used for ASTM and the other part for DSC. Each sample examined with ASTM requires 30 meshes to be fabricated and sampled. The percentage of grid depends on the number of times the sample is irradiated and the higher the sample is under the radiation. The DSC performed 2-4 times for each sample. High-lattice samples yielded $0.06 \mathrm{~min}$ start time and the lowest percentage yielded $0.01 \mathrm{~min}$. Table 1 shows the results. Indicates deviations in ASTM with low and high start times.

Note that for low deflection samples it is, for example, 0.03 minutes, which is probably due to the use of small specimens. Fall. The above data can show that the deviation from the time of reaction onset for the sample that appears to have a constant grid percentage has a maximum of $0.036 \mathrm{~min}$ and a mean of 0.022 min deviation, indicating a maximum error of $10 \%$ and a mean of $6 \%$ and the onset time It is low in percentage. In all the relationships mentioned for the above relationship, the non-networked samples have not been investigated.

The start time for the thermal transition for zero grid percentages was obtained from three data points for minimum percent grid and extrapolated from this second order polynomial graph. In calibration and number deviation were 0.0007 and $0.00011 \mathrm{~min}$, respectively. They are not operational because they are not zero percent and for percentages below 0.5 percent.

\section{RESULT AND DISCUSSION}

As long as there is a direct relationship between the percent of mesh and the melting start time, the calibration curve of the mean start time versus the average gel volume is plotted with the available information. The usual results are shown in Figure 2. Several different sentences were used to fit the data, the lowest order being 3 for mathematical citizenship. The full diagram shown in Figure 2 relates to the data in Table 1 and to their fit with the degree 3 equation. Next is the polyethylene filler data, when this data is plotted, the volume of the gel varies with the start time. The upper curve is higher. This effect was corrected by gel and non-gel samples, probably due to additives such as fillers, which is given in Table 2 and Curve 2. Fit with grade 3 citizenship in these samples is also a good approximation for Find the volume of the gel in the samples. This process is reproducible until two batches of gels match. The diagram for combining the two curves is shown in Fig. 3, along with the fit parameters. This graph contains errors related to the measurement of gel volume in ASTM technique and the time associated with it.

Description of Experiment Theory:

The DSC data show that the heat required for melting to transition from solid to liquid is constant at $4 \%$ and does not show a one-to-one citizenship of the gel volume.

The temperatures that occur during this transition are usually in the $\mathrm{X}+\mathrm{Y}$ degree. After decreasing the start time with the gel volume is a traditional effect, which is obtained by comparing the maximum flow temperature with the field start time ratio (Fig. 1). It is lower in gel percentage than in non-gel samples and shows a nonlinear relationship with the starting time. The shape of the heat versus time graph for samples 
with higher gel content is shorter and shorter than gelless samples. Lower.

Consider the example above with an X-gel fraction and an Xgel-free fraction. They both melt, though at different speeds. At the onset of melting suppose that the gel-forming material begins to deform and, according to the data, it can be said that the material with a high lattice percentage starts to deform earlier than the pure gel-free material, so the relationship between the starting time and The percentages of gels are present. The fusion synthetics are expressed by a relation, the simplest of which is the Avram formula. If we consider fusion and fusion to be similar, we show the percentage of grid with $\mathrm{X}$ and time with $\mathrm{t}$. Given that $\ln (1-\mathrm{X})=-\mathrm{Zt} \mathrm{t}^{\wedge} \mathrm{n}$. If $\mathrm{X}=0$ then $t=0$. It is proposed that the graph of $\ln (1-X)$ be linear against $\mathrm{t}^{\wedge} \mathrm{n}$, as shown in Fig. 4. These 2 parameters fit slightly worse than 4 feet Fittings with higher degree polynomials acknowledge being near optimal. Some of the assumptions in this explanation are untested, but we examined the effects of the heating rate and the melting rate on this system.

\section{CONCLUSION}

The DSC technique was used to measure the gel volume in cross-linked polyethylene. This technique is precisely the standard ASTM technique (ASTM D 2765-01) used to measure the gel volume of different materials. However, the technique presented in this paper is faster, without the use of hazardous materials, and with less manpower and labor than the ASTM method.

\section{REFERENCES}

[1] Ourang, A., Pilehvar, S., Mortezaei, M. and Damircheli, R., 2017. Effect of aluminum doped iron oxide nanoparticles on magnetic properties of the polyacrylonitrile nanofibers. Journal of Polymer Engineering, 37(2), pp.135-141.

[2] Qiu, Q. and Kumosa, M., 1997. Corrosion of E-glass fibers in acidic environments. Composites Science and Technology, 57(5), pp.497-507.

[3] JALALI, Z., HADDADI, A.V., Shabani, I., Soleimani, M., Shafiee, A. and Damircheli, R., 2014. Improving biocompatibility of the polyacrylonitlie nanofibrous scaffolds.

[4] Stockhorst, H. and Brückner, R., 1982. Structure sensitive measurements on E-glass fibers. Journal of noncrystalline solids, 49(1-3), pp.471-484.

[5] JALALI, Z., HADDADI, A.V., Shabani, I., Soleimani, M. and Damircheli, R., 2014. A novel method for producing gelatin nanofibers contains hydroxyapatite nanoparticles.

[6] Jones, R.L., 2006. Chemical Corrosion of E-Glass Fibers in Oxalic and Other Organic Acids. Journal of the American Ceramic Society, 89(1), pp.20-23.

[7] Damircheli, D. and Bhatia, M., 2019. Solution Approaches and Sensitivity Analysis of Variational Inequalities. In AIAA Scitech 2019 Forum (p. 0977).

[8] Benmokrane, B., Wang, P., Ton-That, T.M., Rahman, H. and Robert, J.F., 2002. Durability of glass fiberreinforced polymer reinforcing bars in concrete environment. Journal of Composites for Construction, 6(3), pp.143-153.

[9] Sescu, A., Taoudi, L., Afsar, M.Z. and Thompson, D.S., 2016. Control of Gortler vortices by means of staggered surface streaks. In 46th AIAA Fluid Dynamics Conference (p. 3950)

[10] Velpari, V., PPG Industries Ohio Inc, 2003. Use of Eglass fibers to reduce plastic shrinkage cracks in concrete. U.S. Patent 6,582,511.

[11] Damircheli, R., Mirzadeh, H., Moradi, H., Ghazizadeh, Y. And Jalali, Z., 2014. Electrospun Nanofibrous Scaffolds Based On Alginate For Skin Tissue Engineering.

[12] Damircheli, R., Mirzadeh, H., Moradi, H., Ghazizadeh, Y. And Jalali, Z., 2014. Electrospun Nanofibrous Scaffolds Based On Chitosan For Skin Tissue Engineering. 